\title{
Does gum chewing affect the timing of return of intestinal motility after elective cesarean section?
}

\author{
Atef M. Darwish ${ }^{1}$, Tarek A. Farghly ${ }^{1}$, Beshoy T. Gad ${ }^{2}$, Ahmed M. Abbas ${ }^{1 *}$
}

\begin{abstract}
${ }^{1}$ Department of Obstetrics and Gynecology, Woman's Health Hospital, Faculty of Medicine, Assiut University, Assiut, Egypt

${ }^{2}$ Department of Obstetrics and Gynecology, Assiut Hospital for Obstetrics, Gynecology and Pediatrics, Assiut, Egypt
\end{abstract}

Received: 10 November 2018

Accepted: 06 December 2018

*Correspondence:

Dr. Ahmed M. Abbas,

E-mail: bmr90@hotmail.com

Copyright: () the author(s), publisher and licensee Medip Academy. This is an open-access article distributed under the terms of the Creative Commons Attribution Non-Commercial License, which permits unrestricted non-commercial use, distribution, and reproduction in any medium, provided the original work is properly cited.

\begin{abstract}
Background: The aim is to evaluate the effect of gum chewing on the return of intestinal motility after elective CS. Methods: Study period was from September 2017 to March 2018 at the Assiut Women Health University Hospital. The study was registered as a prospective cohort study (Clinicaltrial.gov NCT03355378). Women planned for elective CS according to selection criteria randomized to two groups: Group 1: included 100 patients who received intraoperative and postoperative non-sugary gum chewing as 15 minutes every 2 hours post-operatively for 6 hours with regular care and Group 2: included 100 patients who received regular care without gum chewing.

Results: No statistically significant difference regarding the baseline criteria of both groups. There was a statistically significant lower systolic and diastolic blood pressure in gum chewing group. Hospital stay of gum chewing group was $7.33 \pm 0.73$ hours versus in non-gum chewing group $20.28 \pm 9.92(\mathrm{p}=0.000)$. Passage of flatus of gum chewing group was $8.54 \pm 0.98$ hours versus in non-gum chewing group $13.22 \pm 3.75(\mathrm{P}=0.000)$.

Conclusions: Gum chewing during elective CS improves gut motility in a safe way resulting in early passage of flatus, less hospital stay, and minimal pain score less cost on hospitals.
\end{abstract}

Keywords: Cesarean section, Gum chewing, Intestinal motility

\section{INTRODUCTION}

Cesarean section (CS) is a major operation frequently performed operation in modern practice. After a steady increase since 1996, the rate of cesarean section leveling at $32.8 \%$ of deliveries. ${ }^{1}$ Considering cesarean section is one of the most common surgeries for women in the world. CS like all surgeries is not without postoperative complications that can lead to increase d length of stay. Patients after abdominal surgery remained inpatient for pain control, management of mechanical factors such as drains and wound vacuums, infection and postoperative ileus. The costs attributed to ileus predominantly involve increased length of stay as patients are not able to return home without confirmed return of bowel function.
Elongated stay and increased expense for the patient while decrease their overall satisfaction and quality of life. ${ }^{2}$ This may also contribute to dissatisfaction with overall surgical outcome and interference with maternal bonding with newborn, not feeling well and are not ambulating, DVT risk increases as well as likelihood of infection from atelectasis leading to pneumonia. ${ }^{3}$

Trials to minimize postoperative stay may include type of anesthesia. Anesthesia used during surgery plays a large role when treating the expecting mother perioperatively. Depending on the country and protocols that are in place, a woman may be sectioned under general anesthesia or regional epidural with an opioid. ${ }^{4}$ Few studies have demonstrated that using epidurals with local anesthetics 
block the afferent and efferent reflex pathways, increasing splanchnic blood flow to the colon resulting in a reduction of the sympathetic cascade having an antiinflammatory effect on the large bowel helping to prevent ileus. ${ }^{5}$ Avoiding opioids and general anesthesia, is a safe and effective method in reducing gastrointestinal dysmotility. ${ }^{2}$ Moreover, post-operative and intraoperative antibiotics are used to prevent infection. The use of postoperative non-steroidal anti-inflammatory drugs (NSAIDs) is often recommended to avoid the dysmotility effects of opiates. ${ }^{6}$

What's new in this era is the concept of "Fast-tract surgery" to obtain enhanced recovery after CS surgery. It aims to hasten functional recovery and improves postoperative outcomes. ERAS programs are usually associated with shorter length of hospital stay, a reduction in overall healthcare costs, and improvements in patient satisfaction. It comprises pre-admission counseling, avoidance of preoperative bowel preparation, use of opioid-sparing multimodal perioperative analgesia (including loco-regional analgesia), intraoperative goaldirected fluid therapy (GDT), and use of minimally invasive surgical techniques. Postoperatively, it is important to encourage early feeding, early mobilization, timely removal of tubes and drains, if present, and function oriented multimodal analgesia regimens. ${ }^{7}$

The theory behind gum chewing is that it mimics feeding and has been considered a form of sham feeding, encouraging the gastro-colic reflex and commencement of the cephalic phase of digestion improving gut motility in a safe way. ${ }^{8}$ There have been some strides in understanding the etiology of POI along with its prevention and management. Gum chewing focuses more on the neurogenic component in trying to support the Migrating Motor Complex in fasting state and simulate more strong peristaltic contractions representative of a fed state. ${ }^{7}$ It has been studied in different parts of the world and was proved to minimize postoperative stay according to passage of flatus.

The current study aims to evaluate the effect of gum chewing on the return of intestinal motility after elective CS.

\section{METHODS}

The Study period from September 2017 to March 2018 at the Assiut Women Health University Hospital . Faculty of medicine ethical committee approved this study on $1 / 11 / 2016$ with no. 17100516 . The study was registered as a prospective cohort study (Clinicaltrial.gov NCT03355378). The collection of cases from women planned for elective CS according to selection criteria.

\section{Inclusion criteria}

- Woman is sure of her dates or documented early pregnancy ultrasound.
- Women aged 20-35 years.

- Women with BMI 18-22 kg/m2.

- Estimated gestational age is ranging between 37-40 weeks gestation.

- Accepting to participate in the study.

\section{Exclusion criteria}

- Women aged $<20$ and $>35$ years

- Caesarean hysterectomy.

- Surgical management of severe postpartum hemorrhage

- History of bowel obstruction, inflammatory bowel disease

- Previous abdominal or pelvic radiation.

All pregnant women were subjected to careful and detailed history and examination.

\section{History}

- Personal history: It included name, age, occupation, residence and special habits of medical importance.

- Obstetric history: It included the first day of last menstrual period for accurate estimation of gestational age and antenatal care.

- Past history: It included the history of any medical disorder or surgical history.

- History of present illness: It included the medical and surgical condition to define high risk pregnancy

\section{Examination}

General examination: It included pulse, blood pressure, temperature and respiratory rate.

Abdominal examination

\section{Investigations}

\section{Ultrasound examination}

- Assess viability of pregnancy, determine gestational age and assess amniotic fluid volume

- Complete blood count and blood group

\section{Sample size calculation}

A power calculation estimated that in order to detect on effect size of 6 hours of difference between means of two independent groups, with a p-value $<0.05$ and $80 \%$ power, confidence level 0.95 , a sample size of 100 women for each group was needed. This calculated using $\mathrm{G}$ power 3.1 program

\section{Randomization}

It was done using computer generated randomization sheet using MedCalc. Version13. 


\section{Patients were classified into two groups}

Group 1: Included 100 patients who received intraoperative and postoperative non-sugary gum chewing as 15 minutes every 2 hours post-operatively for 6 hours with regular care

Group 2: Included 100 patients who received regular care without gum chewing as following:

- $\quad$ Stopped feeding 12 hours before operation

- Stopped fluid 6 hours before operation

- Received low residue diet 3 days before operation

- Received 500 cc saline as pre-operative load by I.V cannula

- $\quad$ Received intra operative $1000 \mathrm{cc}$ saline.

- Received intra and post-operative antibiotics

- Received 3 liters fluids in post-operative 24 hours.

\section{Post-operative pain score}

Using visual analogue scale which is intended to help patient care providers to assess pain according to individual patient needs by using $0-10$ scale for patients depending on their faces or behavioural observation to interpret expressed pain when patient can't communicate her pain intensity as following:

- 0: no pain

- 1-3: feel some pain or discomfort but can complete most activities.

- 4-6: pain makes it different to concentrate and may interfere with your ability to do certain normal activities such as reading and watching $\mathrm{T} \mathrm{V}$.

- 7-9: pain is quite intense and is causing you to avoid or limit physical activity .cannot concentrate on anything except pain.

- 10: worst pain imaginable.

\section{Secondary postoperative follow up}

After one-week cases came to outpatient clinics of women health hospital for check up on operation, counselling for family planning, first passage of flatus and asking them about their satisfaction.

\section{Statistical analysis}

The data were collected and entered onto Microsoft access database to be analyzed using the Statistical Package for Social Science (SPSS Inc., Chicago, version 21). A comparison between two groups was done using Student's t-test to compare the mean values between groups in scale variables. However, Chi 2 test was used to compare the dichotomous and ordinal variables in the groups. For analysis $\mathrm{P}<0.05$ was considered significant. For non-parametric continuous or categorical data, suitable tests as Fisher exact and Man-Whitney tests were used.

\section{RESULTS}

No statistically significant difference regarding the baseline criteria of both groups (Table 1).

Table 1: The baseline characteristics of the study participants.

\begin{tabular}{|c|c|c|c|}
\hline & $\begin{array}{l}\text { Gum } \\
\text { Chewing } \\
(n=100)\end{array}$ & $\begin{array}{l}\text { Without gum } \\
\text { chewing } \\
(\mathrm{n}=100)\end{array}$ & $\begin{array}{l}\text { P- } \\
\text { value }\end{array}$ \\
\hline \multicolumn{3}{|l|}{ Age (years) } & \multirow{3}{*}{0.864} \\
\hline Mean \pm SD & $27.78 \pm 4.88$ & $27.64 \pm 4.79$ & \\
\hline Range & $20.0-35.0$ & $20.0-35.0$ & \\
\hline \multicolumn{3}{|l|}{ Occupation } & \multirow{3}{*}{$0.36 \mathrm{C}$} \\
\hline Working & $4(4.0 \%)$ & $1(1.0 \%)$ & \\
\hline Not working & $96(96.0 \%)$ & $99(99.0 \%)$ & \\
\hline \multicolumn{3}{|l|}{ Residence } & \multirow{3}{*}{0.082} \\
\hline Rural & $39(39.0 \%)$ & $28(28.0 \%)$ & \\
\hline Urban & $61(61.0 \%)$ & $72(72.0 \%)$ & \\
\hline \multicolumn{3}{|l|}{ Gravity } & \multirow{3}{*}{0.177} \\
\hline Mean \pm SD & $3.50 \pm 2.03$ & $3.86 \pm 2.15$ & \\
\hline Range & $1.0-10.0$ & $1.0-11.0$ & \\
\hline \multicolumn{3}{|l|}{ Parity } & \multirow{3}{*}{0.087} \\
\hline Mean \pm SD & $1.90 \pm 1.70$ & $2.33 \pm 1.93$ & \\
\hline Range & $0.0-8.0$ & $0.0-10.0$ & \\
\hline \multicolumn{2}{|c|}{ Gestational age (weeks) } & & \multirow{3}{*}{0.128} \\
\hline Mean \pm SD & $38.29 \pm 1.05$ & $37.93 \pm 0.98$ & \\
\hline Range & $37.0-40.0$ & $37.0-40.0$ & \\
\hline
\end{tabular}

Table 2 shows the vital signs after CS of both groups. There was a statistically significant lower systolic and diastolic blood pressure in gum chewing group.

Table 2: The postoperative vital signs of the study participants.

\begin{tabular}{|c|c|c|c|}
\hline & $\begin{array}{l}\text { Gum } \\
\text { chewing } \\
(n=100)\end{array}$ & $\begin{array}{l}\text { Without gum } \\
\text { chewing } \\
(\mathrm{n}=100)\end{array}$ & $\begin{array}{l}\text { P- } \\
\text { value }\end{array}$ \\
\hline \multicolumn{3}{|l|}{ Pulse } & \multirow{3}{*}{0.404} \\
\hline Mean \pm SD & $79.32 \pm 3.46$ & $79.62 \pm 3.18$ & \\
\hline Range & $73.0-90.0$ & $75.0-90.0$ & \\
\hline \multicolumn{3}{|l|}{ Systolic BP } & \multirow{3}{*}{$0.007 *$} \\
\hline Mean \pm SD & $112.72 \pm 5.63$ & $116.90 \pm 10.98$ & \\
\hline Range & $110.0-140.0$ & $110.0-170.0$ & \\
\hline \multicolumn{3}{|c|}{ Diastolic BP } & \multirow{3}{*}{$0.011^{*}$} \\
\hline Mean \pm SD & $66.15 \pm 5.50$ & $68.50 \pm 6.69$ & \\
\hline Range & $60.0-80.0$ & $60.0-100.0$ & \\
\hline \multicolumn{3}{|c|}{ Temperature } & \multirow{3}{*}{0.157} \\
\hline Mean \pm SD & $37.09 \pm 0.25$ & $37.04 \pm 0.13$ & \\
\hline Range & $36.8-39.0$ & $37.0-38.0$ & \\
\hline
\end{tabular}

On the other hand, no difference regarding the pulse and temperature between both groups. According to Table 3 of study outcomes, it was founded that hospital stay of gum chewing group was: from 6-8 hrs with mean \pm SD $7.33 \pm 0.73$, of non-gum chewing group was from 10$72 \mathrm{hrs}$. 
With mean \pm SD $20.28 \pm 9.92$ with statistically significant difference more in non-gum chewing group $(\mathrm{p}=0.000)$.

Passage of flatus of gum chewing group was: from 6-11 hrs with mean \pm SD $8.54 \pm 0.98$, of non-gum chewing group was: from 9-40 hrs with mean \pm SD 13.22 \pm 3.75 with statistically significant difference more in non-gum chewing group $(\mathrm{P}=0.000)$.

Pain score of gum chewing group was: from 1-3 with mean \pm SD $1.46 \pm 0.54$, of non-gum chewing group was: from 2-3 with mean \pm SD $2.51 \pm 0.050$ with statistically significant difference more in non-gum chewing group. All patients of the two groups had their wounds healed after 1 week of operation with no complications.

Table 3: The study outcomes among the study participants.

\begin{tabular}{|c|c|c|c|}
\hline & $\begin{array}{l}\text { Gum } \\
\text { chewing } \\
(n=100)\end{array}$ & $\begin{array}{l}\text { Without gum } \\
\text { chewing } \\
(\mathrm{n}=100)\end{array}$ & $\begin{array}{l}\text { P. } \\
\text { value }\end{array}$ \\
\hline \multicolumn{2}{|c|}{ Hospital stay (hours) } & & \multirow{3}{*}{$0.000 *$} \\
\hline Mean \pm SD & $7.33 \pm 0.73$ & $20.28 \pm 9.92$ & \\
\hline Range & $6.0-8.0$ & $10.0-72.0$ & \\
\hline \multicolumn{2}{|c|}{ Flatus passage (hours) } & & \multirow{3}{*}{$0.000 *$} \\
\hline Mean \pm SD & $8.54 \pm 0.98$ & $13.22 \pm 3.75$ & \\
\hline Range & $6.0-11.0$ & $9.0-40.0$ & \\
\hline \multicolumn{3}{|l|}{ Pain score } & \multirow{3}{*}{$0.000^{*}$} \\
\hline Mean \pm SD & $1.46 \pm 0.54$ & $2.51 \pm 0.50$ & \\
\hline Range & $1.0-3.0$ & $2.0-3.0$ & \\
\hline \multicolumn{3}{|c|}{ External wound healing after 1 week } & \\
\hline Healed & $100(100.0 \%)$ & $100(100.0 \%)$ & \\
\hline
\end{tabular}

\section{DISCUSSION}

Gum chewing has been studied in different parts of the world and was proved to minimize postoperative stay according to passage of flatus. Previous studies reported prolonged postoperative stay despite gum chewing that reached up to 24-34 hours in other studies. ${ }^{9-12}$ Postoperative pain that reached 3-4 pain score in some studies. ${ }^{9-12}$

This study tested the effect of intraoperative and postoperative gum chewing on ERAS compared to the regular postoperative care of CS at the Woman's Health University Hospital, Assiut, Egypt. We found that gum chewing intra-operatively and postoperatively decreased hospital stay after CS to 6-8 hours in comparison to 10-72 hours without gum chewing. Early passage of flatus with gum chewing within 6-11 hours in comparison to 9-40 hours without gum chewing. Marked decrease in pain score with gum chewing 1-3 in comparison to 2-3 without gum chewing.

ERAS aims to hasten functional recovery and improve post-operative outcomes. ERAS programs are usually associated with shorter length of hospital stay, a reduction in overall healthcare costs, and improvements in patient satisfaction. It comprises preadmission counseling, avoidance of preoperative bowel preparation, use of opioid-sparing multimodal perioperative analgesia (including loco-regional analgesia), intraoperative goaldirected fluid therapy (GDT), and use of minimally invasive surgical techniques with avoidance of routine use of nasogastric tube, drains and/or catheters.

Postoperatively, it is important to encourage early feeding, early mobilization, timely removal of tubes and drains, if present and function oriented multimodal analgesia regimens. ${ }^{13}$ So it is very important in CS to improve bowel motility, earlier passage of flatus, less hospital stay and more patient satisfaction.

The literature indicates that gum-chewing was beneficial in preventing postoperative ileus in post-cesarean section patients. $^{8}$ However, further research is necessary to establish the role of gum-chewing as part of the protocol for patients. This study was not without limitations; however, advanced consideration was likely diminishing their impact. One limitation in conducting this study is fulfilling a sample size with elective or planned cesarean section patients who meet the specific inclusion criteria. The study is not considering patients who first had a trial of labor and transitioned to cesarean. This was an elective CS patient trial only.

Another limitation to this study is the design does not lend itself to blinded participants. Patients in the gum chewing group were clearly be aware of their assignment as well as those in the non-chewing group given all candidates provided consent ahead of time to be allocated to either grouping. The study does permit the nursing staff responsible for recording the data to be blinded as the patients were instructed to not discuss their assignments with medical staff to avoid any bias. Although early feeding protocol was initiated, there were variable results among patients return to eating in conjunction with gum-chewing which may complicate the ability to declare gum chewing as an independent factor. Variability in patient's threshold to pain and request for analgesics or anti-emetics in the postoperative setting was also pose a challenge in discerning definitive data for support of gum chewing; however, these medications were not be controlled by study due to ethical standards. Lastly, this study largely relies on selfreported measurements and the accuracy of participants documenting their bowel function. This factor was hopefully overcome with around the clock reminding from nurses.

\section{CONCLUSION}

Gum chewing is similar to feeding and has been considered a form of sham feeding and proposed to appease thirst, hunger and prevent ileus symptoms in intra and early postoperative period when early feeding is not likely tolerated after anesthesia by encouraging the 
gastro-colic reflex and commencement of the cephalic phase of digestion improving gut motility in a safe way resulting in early passage of flatus, less hospital stay and minimal pain score less cost on hospitals.

Funding: No funding sources

Conflict of interest: None declared

Ethical approval: The study was approved by the Institutional Ethics Committee

\section{REFERENCES}

1. Hamilton BE, Martin JA, Ventura SJ. Births: preliminary data for 2010. Natl Vital Stat Rep. 2011; 60(2):1-25.

2. Holte K, Kehlet H. Postoperative ileus: a preventable event. Brit J Surg. 2002; 87(11):1480-93.

3. Izbizky GH, Minig L, Sebastiani MA, Otano L. The effect of early versus delayed post caesarean feeding on women's satisfaction: a randomized controlled trial. BJOG. 2008;115(3):332-338.

4. Wattwil M, Thoren T, Hennerdal S, Garvill JE. Epidural analgesia with bupivacaine reduces postoperative paralytic ileus after hysterectomy. Anesthes Analges. 2006;68(3),353-8.

5. Luckey AM, Livingston, EM, Tache, YP. Mechanisms of postoperative ileus. Arch Surg. 2003;138(2):206-14.

6. Doorly M, Senagore A. Pathogenesis and clinical and economic consequences of postoperative ileus. Surg Clinic North Am. 2012;92(2):259-72.

7. Johnson MD, Walsh RM. Current therapies to shorten postoperative ileus. Cleveland Clinic J Med. 2009;76(11):641-8.
8. Castro SMM de, van den Esschert JW, van Heek NT, Dalhuisen S, Koelemay MJW, et al. A systematic review of efficacy of gum chewing for the amelioration of postoperative ileus. Digest Surg. 2008;25(1):39-45.

9. Abd-El-Maeboud, K H I, Ibrahim M I, Shalaby DAA, Fikry M F. Gum chewingstimulates early return of bowel motility after caesarean section. Int $\mathbf{J}$ Obstet Gynecol. 2009;116(10):1334-39.

10. Ledari F M, Barat S, Delavar M A. Chewing gum has stimulatory effects on bowel function in patients undergoing cesarean section: A randomized controlled trial. Bosn J Basic Med Sci. 2012;12(4): 265-268.

11. Tandeter H. Hypothesis: hexitols in chewing gum may play a role in reducing postoperative ileus. Medical Hypothes. 2009;72(1):39-40.

12. Jakkaew B, Charoenkwan K. Effects of gum chewing on recovery of bowel function following cesarean section: a randomized controlled trial. Arch Gynecol Obstet. 2013; 288(2):255-60.

13. Shang H, Yang Y, Tong X, Zhang L, Fang A, Hong L. Gum chewing slightly enhances early recovery from postoperative ileus after cesarean section: results of a prospective, randomized, controlled trial. Am J Perinatol.2009;27(05):387-91.

Cite this article as: Darwish AM, Farghly TA, Gad BT, Abbas AM. Does gum chewing affect the timing of return of intestinal motility after elective cesarean section?. Int J Reprod Contracept Obstet Gynecol 2019;8:290-4. 